\title{
SINGULAR LOCI OF SCHUBERT VARIETIES FOR CLASSICAL GROUPS
}

\author{
V. LAKSHMIBAI
}

In this note, we give an explicit description of the singular locus of a Schubert variety in the flag variety $G / B$, where $G$ is a classical group, and $B$ a Borel subgroup of $G$.

Let $G$ be a classical group, and $T$ a maximal torus in $G$. Let $W$ be the Weyl group, and $R$ the system of roots, of $G$ relative to $T$. Let $B$ be a Borel subgroup of $G$, where $B \supset T$. Let $S$ (resp. $R^{+}$) be the set of simple (resp. positive) roots of $R$ relative to $B$. For $\alpha \in R$, let $s_{\alpha}$ be the reflection with respect to $\alpha$, and $X_{\alpha}$ the element in the Chevalley basis for the Lie algebra of $G$, associated to $\alpha$. For $w \in W$, let $e(w)$ denote the point in $G / B$ corresponding to $w$. The Schubert variety $X(w)$, where $w \in W$, is by definition the Zariski closure of $B e(w)$ in $G / B$. $(X(w)$ is understood to be endowed with the canonical reduced structure.) Let $\succeq$ denote the Bruhat order in $W$. It is well known that for $w_{1}, w_{2} \in W$,

$$
w_{1} \succeq w_{2} \text { if and only if } X\left(w_{1}\right) \supseteq X\left(w_{2}\right) .
$$

(For generalities on algebraic groups, one may refer to $[\mathbf{1}]$.)

The results on the singular locus of a Schubert variety are obtained as consequences of "standard monomial theory" as developed in Geometry of $G / P$. I-V (cf. $[11,7,4,5,8])$. One of the consequences of standard monomial theory is the First Basis Theorem (cf. $[\mathbf{5}, \mathbf{8}, \mathbf{6}]$ ) which gives a $\mathbf{Z}$ basis

$$
\{P(\lambda, \mu),(\lambda, \mu) \text { an admissible pair }\}
$$

for $H^{0}\left(G_{\mathbf{z}} / P_{\mathbf{z}}, L_{\mathbf{Z}}\right)$, where $P_{\mathbf{Z}}$ is a maximal parabolic subgroup scheme of $G_{\mathbf{Z}}$ and $L_{\mathbf{Z}}$ is the ample generator of $\operatorname{Pic}\left(G_{\mathbf{Z}} / P_{\mathbf{Z}}\right)$. For any field $k$, let us denote the canonical image of $P(\lambda, \mu)$ in $H^{0}\left(G_{\mathbf{Z}} \otimes k / P_{\mathbf{Z}} \otimes k, L_{\mathbf{Z}} \otimes k\right)$ by $p(\lambda, \mu)$. In [9], it is shown that over any field $k$, for $w, \tau \in W$, with $w \succeq \tau$, the Zariski tangent space $T(w, \tau)$, to $X(w)$ at $e(\tau)$ is spanned by

$$
\left\{\begin{array}{l|l}
X_{-\beta}, \beta \in \tau\left(R^{+}\right) & \begin{array}{l}
\text { for all }(\lambda, \mu) \text { such that } X_{-\beta} p(\lambda, \mu)=c p(\tau, \tau), c \in k^{*}, \\
\left.p(\lambda, \mu)\right|_{X(w)} \neq 0
\end{array}
\end{array}\right\} .
$$

Denoting by $\{Q(\lambda, \mu)\}$ the basis for the Z-dual of $H^{0}\left(G_{\mathbf{Z}} / P_{\mathbf{Z}}, L_{\mathbf{Z}}\right)$, dual to the basis $\{P(\lambda, \mu)\}$, it can be seen easily that $X_{-\beta} p(\lambda, \mu)=c p(\tau, \tau), c \in k^{*}$, if and only if $X_{-\beta} Q(\tau, \tau)$, when written as a Z-linear combination of the elements

Received by the editors June 7, 1985 and, in revised form, May 14, 1986.

1980 Mathematics Subject Classification (1985 Revision). Primary 14M15; Secondary $20 \mathrm{G05}$.

Research partially supported by the NSF. 
$Q(\theta, \delta)$, involves $Q(\lambda, \mu)$ with a coefficient that is nonzero in $k$. From this we obtain that $T(w, \tau)$ is spanned by

$$
\left\{\begin{array}{l|l}
X_{-\beta}, \beta \in \tau\left(R^{+}\right) & \begin{array}{l}
\text { for all }(\lambda, \mu) \text { such that } Q(\lambda, \mu) \text { occurs in } X_{-\beta} Q(\tau, \tau) \\
\text { with a coefficient that is nonzero in } k, w \succeq \lambda
\end{array}
\end{array}\right\} .
$$

In [3], we have given an explicit description of $Q(\lambda, \mu)$ for the case of a classical group. Using this description, we express $X_{-\beta} Q(\tau, \tau)$ as a linear combination with integer coefficients of the $Q(\theta, \delta)$ 's. This enables us to obtain an explicit description of the singular locus of $X(w)$.

Let $G$ be classical of rank $n$. Let $S=\left\{\alpha_{1}, \ldots, \alpha_{n}\right\}$, the order being as in [2]. Further, we follow the notation in [2] to denote the elements of $R$. For $1 \leq d \leq n$, we fix the following:

$$
\begin{aligned}
P_{d} & =\left\{\begin{array}{l}
\text { the maximal parabolic subgroup of } G \\
\text { obtained by "omitting the simple root } \alpha_{d}
\end{array}\right. \\
W_{P_{d}} & =\text { Weyl group of } P_{d}, \\
W^{P_{d}} & =\text { the set of "minimal representatives" of } W / W_{P_{d}} .
\end{aligned}
$$

Recall (cf. $[2,4])$ that

$$
W^{P_{d}}=\left\{w \in W \mid l\left(w s_{\alpha_{i}}\right)=l(w)+1,1 \leq i \leq n, i \neq d\right\} .
$$

It is known (cf. [2]) that

$$
W=W_{P_{d}} \cdot W^{P_{d}} .
$$

For $w \in W$, let $w^{(d)}$ be the element in $W^{P_{d}}$ corresponding to the coset $w W_{P_{d}}$. We have

$$
w^{(d)}=w W_{P_{d}} \cap W^{P_{d}} .
$$

Let

$$
A=\left\{\left(a_{1}, \ldots, a_{d}\right) \mid a_{1}<a_{2}<\cdots<a_{d}, a_{i} \in \mathbf{Z}\right\} .
$$

We have a natural partial order $\geq$ in $A$, namely,

$$
\left(a_{1}, \ldots, a_{d}\right) \geq\left(b_{1}, \ldots, b_{d}\right), \quad \text { if } a_{i} \geq b_{i}, 1 \leq i \leq d .
$$

This partial order among $d$-tuples will be used in the sequel in describing the Bruhat order in $W^{P_{d}}$. Further, for any $d$-tuple $\left(z_{1}, \ldots, z_{d}\right)$ of integers, we let

$$
\left(z_{1}, \ldots, z_{d}\right) \uparrow=\left(z_{i_{1}}, z_{i_{2}}, \ldots, z_{i_{d}}\right)
$$

where $j \rightarrow i_{j}$ is a permutation and $z_{i_{j}} \leq z_{i_{j+1}}$. Thus, $\left(z_{1}, \ldots, z_{d}\right) \uparrow$ is the $d$-tuple whose entries are obtained by arranging the entries $\left(z_{1}, \ldots, z_{d}\right)$ in increasing order. We shall denote the elements of the symmetric group $S_{m}$, where $m \in \mathbf{N}$, in the following way. Let $\sigma \in S_{m}$ be such that

$$
\sigma(i)=c_{i}, \quad 1 \leq i \leq m .
$$

We shall denote $\sigma$ by $\left(c_{1} \cdots c_{m}\right)$. Let $k$ be the base field. For any positive integer $m$, let $\left\{e_{1}, \ldots, e_{m}\right\}$ denote the standard basis of $k^{m}$. 
I. The symplectic group $\operatorname{Sp}(2 n)$. Let $E=\left(\begin{array}{cc}0 & J \\ -J & 0\end{array}\right)$, where

$$
J=\left(\begin{array}{ccc}
0 & & 1 \\
& . \cdot & \\
1 & & 0
\end{array}\right)_{n \times n} .
$$

Let $($,$) be the skew symmetric bilinear form on k^{2 n}$, represented by $E$, with respect to $\left\{e_{1}, \ldots, e_{2 n}\right\}$. Let

$$
G=\operatorname{Sp}(2 n)=\left\{A \in \mathrm{SL}(2 n) \mid{ }^{t} A E A=E\right\} .
$$

Let $\sigma$ be the involution on $\mathrm{SL}(2 n)$ defined by

$$
\sigma(A)=E\left({ }^{t} A\right)^{-1} E^{-1}, \quad A \in \operatorname{SL}(2 n) .
$$

We see that

$$
\mathrm{Sp}(2 n)=\mathrm{SL}(2 n)^{\sigma} \text {. }
$$

In view of (8), we obtain an identification of $W$, the Weyl group of $G$, with a subgroup of $S_{2 n}$ (= the Weyl group of $\mathrm{SL}(2 n)$ ), namely

$$
W=\left\{\left(a_{1} \cdots a_{2 n}\right) \mid a_{i}=2 n+1-a_{2 n+1-i}, 1 \leq i \leq 2 n\right\} .
$$

See [7] for details.

The above identification (cf. (9)) of $W$, and straightforward calculations using the definitions of [2] allow us to identify $W^{P_{d}}$ as

$$
W^{P_{d}}=\left\{\begin{array}{l|l}
\left(a_{1}, \ldots, a_{d}\right) & \begin{array}{l}
\text { (1) } 1 \leq a_{1}<a_{2}<\cdots<a_{d} \leq 2 n, \\
\text { (2) for } 1 \leq i \leq 2 n, \text { if } i \in\left\{a_{1}, \ldots, a_{d}\right\} \\
\text { then } 2 n+1-i \notin\left\{a_{1}, \ldots, a_{d}\right\}
\end{array}
\end{array}\right\}
$$

For $w \in W$, say $w=\left(c_{1} \cdots c_{2 n}\right)$, we see easily that

$$
w^{(d)}=\left(c_{1}, \ldots, c_{d}\right) \uparrow .
$$

Under the above identification of $W^{P_{d}}$, we have (cf. [10]), given two elements $\left(a_{1}, \ldots, a_{d}\right),\left(b_{1}, \ldots, b_{d}\right)$ in $W^{P_{d}}$,

(12) $\left(a_{1}, \ldots, a_{d}\right) \succeq\left(b_{1}, \ldots, b_{d}\right)$ if and only if $\left(a_{1}, \ldots, a_{d}\right) \geq\left(b_{1}, \ldots, b_{d}\right)$.

Thus, the Bruhat order in $W^{P_{d}}$ coincides with the natural order (cf. equation (3)) on $d$-tuples.

Proposition C.1. Let $G=\operatorname{Sp}(2 n)$. For $1 \leq i \leq 2 n$, let $i^{\prime}=2 n+1-i$ and $|i|=\min \left\{i, i^{\prime}\right\}$. Let $w, \tau \in W$, with $w \succeq \tau$. Let $\tau=\left(a_{1} \cdots a_{2 n}\right)$. Then the tangent space $T(w, \tau)$ to $X(w)$ at $e(\tau)$ is spanned by the set of root vectors $\left\{X_{-\beta}, \beta \in N(w, \tau)\right\}$, where $N(w, \tau)$ is the subset of $\tau\left(R^{+}\right)$consisting of roots $\beta$ which satisfy criteria (a) and (b) below. Let $\beta=\tau(a), \alpha \in R^{+}$. We follow the notation of [2] for elements of $R^{+}$.

(a) Let $\alpha=\varepsilon_{j}-\varepsilon_{k}, 1 \leq j<k \leq n$ or $2 \varepsilon_{j}, 1 \leq j \leq n$. Then

$$
w \succeq s_{\beta} \tau \text {. }
$$


(b) Let $\alpha=\varepsilon_{j}+\varepsilon_{k}, 1 \leq j<k \leq n$. Let $s\left(\right.$ resp. r) be the $\min \left\{\left|a_{j}\right|,\left|a_{k}\right|\right\}$ (resp. $\left.\max \left\{\left|a_{j}\right|,\left|a_{k}\right|\right\}\right)$. Then

$$
w^{(j)} \succeq\left(a_{1}, \ldots, a_{j-1}, a_{k}^{\prime}\right) \uparrow
$$

and

$$
w^{(k)} \succeq\left(a_{1}, \ldots, \hat{a}_{j}, \ldots, a_{k-1}, r, s^{\prime}\right) \uparrow .
$$

II. The special orthogonal group $\operatorname{So}(2 n+1)$. Let

$$
E=\left(\begin{array}{lll}
0 & & 1 \\
& . & \\
1 & & 0
\end{array}\right)_{2 n+1 \times 2 n+1},
$$

and let $($,$) be the symmetric bilinear form on k^{2 n+1}$, respresented by $E$, with respect to $\left\{e_{1}, \ldots, e_{2 n+1}\right\}$. Let

$$
G=\operatorname{So}(2 n+1)=\left\{\left.A \in \mathrm{SL}(2 n+1)\right|^{t} A E A=E\right\} .
$$

Let $\sigma$ be the involution on $\mathrm{SL}(2 n+1)$ defined by

$$
\sigma(A)=E\left({ }^{t} A\right)^{-1} E, \quad A \in \mathrm{SL}(2 n+1) .
$$

As in $§ I$, we have

$$
\operatorname{So}(2 n+1)=\operatorname{SL}(2 n+1)^{\sigma} \text {. }
$$

In view of (15), we obtain identifications for the Weyl group $W$, and also for $W^{P_{d}}$ similar to (9) and (10), namely

(16) $W=\left\{\left(a_{1} \cdots a_{2 n+1}\right) \in S_{2 n+1} \mid a_{i}=2 n+2-a_{2 n+2-i}, 1 \leq i \leq 2 n+1\right\}$

and

$$
W^{P_{d}}=\left\{\begin{array}{l|l}
\left(a_{1}, \ldots, a_{d}\right) & \begin{array}{l}
\text { (1) } 1 \leq a_{1}<a_{2}<\cdots<a_{d} \leq 2 n+1, \\
\text { (2) } a_{i} \neq n+1,1 \leq i \leq d, \\
\text { (3) For } 1 \leq i \leq 2 n+1, \text { if } i \in\left\{a_{1}, \ldots, a_{d}\right\} \\
\text { then } 2 n+2-i \notin\left\{a_{1}, \ldots, a_{d}\right\}
\end{array}
\end{array}\right\}
$$

For $w \in W$, say $w=\left(c_{1} \cdots c_{2 n+1}\right)$, we have

$$
w^{(d)}=\left(c_{1}, \ldots, c_{d}\right) \uparrow .
$$

As in $§ I$, we have (cf. $[\mathbf{1 0}])$ that the Bruhat order in $W^{P_{d}}$ coincides with the natural order (cf. equation (3)) on $d$-tuples.

Proposition B.1. (Assume char $k \neq 2$.) Let $G=\operatorname{So}(2 n+1)$. For $1 \leq i \leq 2 n+1$, let $i^{\prime}=2 n+2-i$ and $|i|=\min \left\{i, i^{\prime}\right\}$. Let $w, \tau \in W$, with $w \succeq \tau$, and let $\tau=\left(a_{1} \cdots a_{2 n+1}\right)$. Then the tangent space $T(w, \tau)$ to $X(w)$ at $e(\tau)$ is spanned by the set of root vectors $\left\{X_{-\beta}, \beta \in N(w, \tau)\right\}$, where $N(w, \tau)$ $i$ the subset of $\tau\left(R^{+}\right)$consisting of roots $\beta$ which satisfy criteria $(\mathrm{a}),(\mathrm{b})$, and (c) below. Let $\beta=\tau(\alpha), \alpha \in R^{+}$.

(a) Let $\alpha=\varepsilon_{j}-\varepsilon_{k}, 1 \leq j<k \leq n$. Then

$$
w \succeq s_{\beta} \tau \text {. }
$$


(b) Let $\alpha=\varepsilon_{j}+\varepsilon_{k}, 1 \leq j<k \leq n$. Let $s$ (resp. $r$ ) be the minimum (resp. maximum) of $\left\{\left|a_{j}\right|,\left|a_{k}\right|\right\}$.

(i) Suppose precisely one of $\left\{a_{j}, a_{k}\right\}$ does not exceed $n$. Then

$$
\begin{aligned}
& w^{(j)} \succeq\left(a_{1}, \ldots, a_{j-1}, a_{k}^{\prime}\right) \uparrow, \\
& w^{(k)} \succeq\left(a_{1}, \ldots, \hat{a}_{j}, \ldots, a_{k-1}, r, s^{\prime}\right) \uparrow,
\end{aligned}
$$

and

$$
w^{(n)} \succeq\left(s_{\beta} \tau\right)^{(n)} .
$$

(ii) Suppose $a_{j}, a_{k}$ either both exceed $n$ or both do not exceed $n$. For $k \leq d \leq n-1$, let $s_{c(d)}$ be the largest integer, $r<s_{c(d)} \leq n$, such that $s_{c(d)} \notin\left\{\left|a_{1}\right|, \ldots,\left|a_{d}\right|\right\}$ (if no such integer exists, we let $s_{c(d)}=r$ ). Then

$$
\begin{aligned}
& w^{(j)} \succeq\left(a_{1}, \ldots, a_{j-1}, a_{k}^{\prime}\right) \uparrow, \\
& w^{(d)} \succeq\left(a_{1}, \ldots, \hat{a}_{j}, \ldots, \hat{a}_{k}, \ldots, a_{d}, s_{c(d)}^{\prime}, s^{\prime}\right) \uparrow, \quad k \leq d \leq n-1,
\end{aligned}
$$

and

$$
w^{(n)} \succeq\left(s_{\beta} \tau\right)^{(n)} .
$$

(c) Let $\alpha=\varepsilon_{j}, 1 \leq j \leq n$. For $j \leq d \leq n-1$, let $s_{m(d)}$ be the largest integer, $\left|a_{j}\right|<s_{m(d)} \leq n$, such that $s_{m(d)} \notin\left\{\left|a_{1}\right|, \ldots,\left|a_{d}\right|\right\}$ (if no such $s_{m(d)}$ exists, we let $\left.s_{m(d)}=\left|a_{j}\right|\right)$. Then

$$
w^{(d)} \succeq\left(a_{1}, \ldots, \hat{a}_{j}, \ldots, a_{d}, s_{m(d)}^{\prime}\right) \uparrow, \quad j \leq d \leq n-1,
$$

and

$$
w^{(n)} \succeq\left(s_{\beta} \tau\right)^{(n)} .
$$

III. The special orthogonal group $\operatorname{So}(2 n)$. Let

$$
E=\left(\begin{array}{lll}
0 & & 1 \\
& . & \\
1 & & 0
\end{array}\right)_{2 n \times 2 n},
$$

and let $($,$) be the symmetric bilinear form on k^{2 n}$, represented by $E$, with respect to $\left\{e_{1}, \ldots, e_{2 n}\right\}$. Let

$$
G=\operatorname{So}(2 n)=\left\{\left.A \in \mathrm{SL}(2 n)\right|^{t} A E A=E\right\} .
$$

Let $\sigma$ be the involution on $\mathrm{SL}(2 n)$ defined by

$$
\sigma(A)=E\left({ }^{t} A\right)^{-1} E, \quad A \in \mathrm{SL}(2 n) .
$$

We have

$$
\operatorname{So}(2 n)=\operatorname{SL}(2 n)^{\sigma} \text {. }
$$

As in $\S \S I$ and II, we obtain, in view of (21), identifications (described below) for $W$ and $W^{P_{d}}$. We have

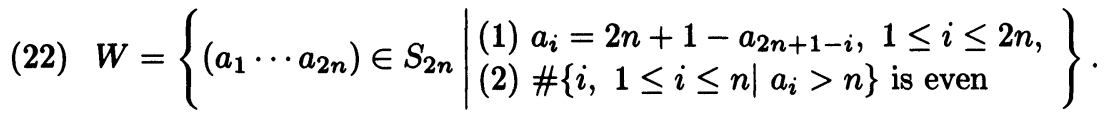


For $1 \leq d \leq n$, let

$$
Z_{d}=\left\{\begin{array}{l|ll}
\left(a_{1}, \ldots, a_{d}\right) & \begin{array}{ll}
(1) & 1 \leq a_{1}<a_{2}<\cdots<a_{d} \leq 2 n \\
(2) & \text { for } 1 \leq i \leq 2 n, \text { if } i \in\left\{a_{1}, \ldots, a_{d}\right\} \\
2 n+1-i \notin\left\{a_{1}, \ldots, a_{d}\right\}
\end{array}, \text { then }
\end{array}\right\}
$$

We have for $d \neq n-1$

$$
W^{P_{d}}=Z_{d}
$$

For $d=n-1$, if $w \in W^{P_{d}}$, then

$$
w \equiv w u_{i}\left(\bmod W_{P_{n-1}}\right), \quad 0 \leq i \leq n, i \neq n-1,
$$

where

$$
u_{i}= \begin{cases}s_{\alpha_{n}} & \text { if } i=n \\ \operatorname{Id} & \text { if } i=0 \\ s_{\alpha_{i}} s_{\alpha_{i+1}} \cdots s_{\alpha_{n-2}} s_{\alpha_{n}} & \text { if } 1 \leq i \leq n-2\end{cases}
$$

(Here Id denotes the identity element in W.) In particular, for $w_{1}, w_{2} \in$ $W$, say $w_{1}=\left(a_{1} \cdots a_{2 n}\right), w_{2}=\left(b_{1} \cdots b_{2 n}\right)$, we can have $w_{1}^{(n-1)}=w_{2}^{(n-1)}$ without $\left(a_{1}, \ldots, a_{n-1}\right) \uparrow$ and $\left(b_{1}, \ldots, b_{n-1}\right) \uparrow$ being the same. Thus $W^{P_{n-1}}$ gets identified with a proper subset of $Z_{n-1}$ (cf. Definition (23)). For $w \in W$, say $w=\left(c_{1} \cdots c_{2 n}\right)$, we have

$$
w^{(d)}=\left(c_{1}, \ldots, c_{d}\right) \uparrow, \quad d \neq n-1 .
$$

To describe $w^{(n-1)}$, we let, for $1 \leq i \leq n, i \neq n-1$,

$$
\left(y_{1}^{(i)}, \ldots, y_{n-1}^{(i)}\right)=\left\{\begin{array}{l}
\text { the }(n-1) \text {-tuple given by the first }(n-1) \\
\text { entries in } w u_{i}
\end{array}\right.
$$

and

$$
Y=\left\{\left(y_{1}^{(i)}, \ldots, y_{n-1}^{(i)}\right) \uparrow, 0 \leq i \leq n, i \neq n-1\right\} .
$$

We observe that $Y$ is totally ordered under $\geq$ (cf. (3)). We have

$$
w^{(n-1)}=\text { the smallest (under } \geq \text { ) element in } Y \text {. }
$$

Unlike the cases of $\operatorname{Sp}(2 n)$ (resp. $\operatorname{So}(2 n+1)$ ), the Bruhat order in $W$, the Weyl group of $\operatorname{So}(2 n)$, is not induced from the Bruhat order in $S_{2 n}$. Hence the Bruhat order in $W^{P_{d}}$ does not coincide with the natural order on $d$-tuples (cf. (3)). We now describe the Bruhat order in $W^{P_{d}}$.

For $1 \leq i \leq 2 n$, let

$$
i^{\prime}=2 n+1-i \text { and }|i|=\min \left\{i, i^{\prime}\right\}
$$

Under the above identification, given two elements $\left(a_{1}, \ldots, a_{d}\right),\left(b_{1}, \ldots, b_{d}\right)$ in $W^{P_{d}}, 1 \leq d \leq n$, we have (cf. [10])

$$
\left(a_{1}, \ldots, a_{d}\right) \succeq\left(b_{1}, \ldots, b_{d}\right)
$$

if and only if the following two conditions hold:
(A) $\left(a_{1}, \ldots, a_{d}\right) \geq\left(b_{1}, \ldots, b_{d}\right)$. 
(B) Suppose for some $r, 1 \leq r \leq d$, and some $i, 0 \leq i \leq d-r$,

$$
\left(\left|a_{i+1}\right|, \ldots,\left|a_{i+r}\right|\right) \uparrow=\left(\left|b_{i+1}\right|, \ldots,\left|b_{i+r}\right|\right) \uparrow=\{n+1-r, \ldots, n\} .
$$

Then

$$
\#\left\{j, i+1 \leq j \leq i+r \mid a_{j}>n\right\}
$$

and

$$
\#\left\{j, i+1 \leq j \leq i+r \mid b_{j}>n\right\}
$$

should both be odd or both even.

Proposition D.1. (Assume char $k \neq 2,3$.) Let $G=\operatorname{So}(2 n)$. Let $w, \tau \in$ $W$, with $w \succeq \tau$, and let $\tau=\left(a_{1} \cdots a_{2 n}\right)$. Then the tangent space $T(w, \tau)$ to $X(w)$ at $e(\tau)$ is spanned by the set of root vectors $\left\{X_{-\beta}, \beta \in N(w, \tau)\right\}$, where $N(w, \tau)$ is the subset of $\tau\left(R^{+}\right)$consisting of roots $\beta$ which satisfy criteria (a) and (b) below. Let $\beta=\tau(\alpha), \alpha \in R^{+}$.

(a) Let $\alpha=\varepsilon_{j}-\varepsilon_{k}, 1 \leq j<k \leq n$. Then

$$
w \succeq s_{\beta} \tau \text {. }
$$

(b) Let $\alpha=\varepsilon_{j}+\varepsilon_{k}, 1 \leq j<k \leq n$. Let $s$ (resp. r) be the minimum (resp. maximum) of $\left\{\left|a_{j}\right|,\left|a_{k}\right|\right\}$.

(i) Suppose precisely one of $\left\{a_{j}, a_{k}\right\}$ does not exceed $n$. Then

$$
\begin{aligned}
& w^{(j)} \succeq\left(a_{1}, \ldots, a_{j-1}, a_{k}^{\prime}\right) \uparrow, \\
& w^{(k)} \succeq\left(a_{1}, \ldots, \hat{a}_{j}, \ldots, a_{k-1}, r, s^{\prime}\right) \uparrow, \\
& w^{(n-1)} \succeq\left(s_{\beta} \tau\right)^{(n-1)},
\end{aligned}
$$

and

$$
w^{(n)} \succeq\left(s_{\beta} \tau\right)^{(n)} .
$$

(ii) Suppose $a_{j}, a_{k}$ either both exceed $n$ or both do not exceed $n$. For $k \leq d \leq n-2$, let $s_{-l(d)}, \ldots, s_{-1}, s_{0}, s_{1}, \ldots, s_{c(d)}$ be the integers

$s<s_{-l(d)}<s_{-l(d)+1}<\cdots<s_{-1}<s_{0}=r<s_{1}<\cdots<s_{c(d)} \leq n$

such that

$$
s_{i} \notin\left\{\left|a_{1}\right|, \ldots,\left|a_{d}\right|\right\}, \quad-l(d) \leq i \leq c(d), \quad i \neq 0 .
$$

Then

$$
\begin{aligned}
& w^{(j)} \succeq\left(a_{1}, \ldots, a_{j-1}, a_{k}^{\prime}\right) \uparrow, \\
& w^{(d)} \succeq \begin{cases}\left(a_{1}, \ldots, \hat{a}_{j}, \ldots, \hat{a}_{k}, \ldots, a_{d}, s_{c(d)-1}^{\prime}, s^{\prime}\right) \uparrow & \text { if }(l(d), c(d)) \neq(0,0), \\
\left(a_{1}, \ldots, \hat{a}_{j}, \ldots, \hat{a}_{k}, \ldots, a_{d}, r^{\prime}, s^{\prime}\right) \uparrow & \text { if }(l(d), c(d))=(0,0),\end{cases} \\
& \quad \text { and for } d=n-1 \text { or } n,
\end{aligned}
$$

$$
w^{(d)} \succeq\left(s_{\beta} \tau\right)^{(d)}
$$




\section{Concluding remarks.}

Corollary. Let $G$ be of type $\mathbf{B}_{n}, \mathbf{C}_{n}$, or $\mathbf{D}_{n}$ and let $w \in W$. Then $X(w)$ is smooth if and only if $\# N(w, \mathrm{Id})=l(w)$, where $N(w, \mathrm{Id})$ is given by Proposition C.1, B.1, or D.1 according as $G$ is of type $C_{n}, B_{n}$, or $D_{n}$, with $\tau=\mathrm{Id}$, the identity element of $W$.

REMARK 1. For $G$ of type $A_{n}$, similar results as above are described in [9].

REMARK 2. Even if char $k=2$ or 3 (in the case of special orthogonal groups), using the explicit computations of $X_{-\beta} Q(\tau, \tau)$, one can still describe $T(w, \tau)$ in a way similar to Propositions B.1 and D.1.

\section{REFERENCES}

1. A. Borel, Linear algebraic groups, W. A. Benjamin, New York, 1969.

2. N. Bourbaki Groupes et algèbres de Lie, Chapitres 4, 5, et 6, Hermann, Paris, 1968.

3. V. Lakshmibai, Bases pour les representations fondamentales des groupes classique. I, II, C. R. Acad. Sci. Paris Sér. I Math. 302, 1986.

4. V. Lakshmibai, C. Musili, and C. S. Seshadri, Geometry of G/P. III, Proc. Indian Acad. Sci. Math. Sci. 88A (1978), 93-177.

5. __ Geometry of G/P. IV, Proc. Indian Acad. Sci. Math. Sci. 88A (1979), 279-362.

6. _ Geometry of $G / P$, Bull. Amer. Math. Soc. (N.S.) 1 (1979), 432-435.

7. V. Lakshmibai and C. S. Seshadri, Geometry of G/P. II, Proc. Indian Acad. Sci. Math. Sci. 87A (1978), 1-54.

8. __ Geometry of G/P. V, J. Algebra 100 (1986), 462-557.

9. __ Singular locus of a Schubert variety, Bull. Amer. Math. Soc. 2 (1984), 363366.

10. R. Proctor, Classical Bruhat orders and lexicographic shellability, J. Algebra 77 (1982), 104-126.

11. C. S. Seshadri, Geometry of G/P. I, C. P. Ramanujan: A Tribute, Tata Inst. Fund. Res. Studies in Math., Springer-Verlag, Berlin, 1978, pp. 207-239.

Department of Mathematics, Texas A\&M University, College Station, TEXAS 77843 